\title{
REGULAÇÃO DE ACESSO À INTERNAÇÃO EM UTI: ANÁLISE PELA TEORIA DA INOVAÇÃ O EM SERVIÇOS ${ }^{1}$
}

\section{ACCESS REGULATION TO ICU: ANALYSIS BY THE THEORY OF INNOVATION IN SERVICES}

\section{REGULACIÓN DEL ACCESO A LA UNIDAD DE CUIDADOS INTENSIVOS: ANÁLISIS BAEADO EN LA TEORÍA DE LA INNOVACIÓN EN LOS SERVICIOS}

\author{
DAGOMAR HENRIQUES LIMA \\ Tribunal de Contas da União \\ dagomarhl@tcu.gov.br
}

\author{
EDUARDO RAUPP VARGAS \\ COPPEAD/Universidade Federal do Rio de Janeiro \\ eduardo.raupp@coppead.ufrj.br
}

\begin{abstract}
RESUMO
Partindo da abordagem integradora da teoria da inovação em serviços, o processo de inovação foi estudado, considerando a regulação de internação em unidades de terapia intensiva (RIUTI) no Distrito Federal como um feixe de cinco serviços elementares constitutivos. Os modos de inovação, as lógicas de serviço e as operações de serviço envolvidas na RIUTI foram descritos nos cinco serviços constitutivos. Baseando-se no conceito de inovação como variações nos componentes de vetores de características dos produtos dos serviços, foi possível identificar a variedade dos processos de inovação nos diferentes serviços e estabelecimentos. O setor público apresentou-se como protagonista do processo de inovação e indutor de inovações no setor privado, necessárias para viabilizar a inserção desses estabelecimentos na RIUTI. Foram constatadas melhorias expressivas na equidade de acesso à internação em unidade de terapia intensiva no Distrito Federal. Permanecem obstáculos estruturais e de gestão que impedem avanços adicionais na integralidade da atenção em saúde. Entre as limitações locais, destacam-se as deficiências no sistema de contrarreferência e na oferta de serviços de média complexidade.
\end{abstract}

PALAVRAS-CHAVE: inovação em serviços; regulação de acesso; sistema único de saúde

\section{ABSTRACT}

Drawing on the integrative approach of the theory of innovation in services, the innovation process to enable the service of access regulation to intensive care units in the Federal District has been studied considering the service as a bundle of five elementary constituent services. The modes of innovation, service logic and service operations involved in the access regulation to intensive care units were described in five constituent services. Based on the concept of innovation as variations in the components of vectors of characteristics of the service products, it was possible to identify the variety of innovative processes in distinct services and organizations. The public sector acted as a protagonist of the innovation process and induced innovations in the private sector. Significant improvements were found in the equity of access. Structural and management barriers remain and prevent further advances in the integrality of health care. Among the local limitations, we highlight the deficiencies in the system of counter-reference and in the provision of medium complexity health services.

KEYWORDS: service innovation; access regulation; unified health system

\section{RESUMEN}

Basándose en el enfoque integrador de la teoría de la innovación en los servicios, se ha estudiado el servicio de la regulación del acceso a las unidades de cuidados intensivos en el Distrito Federal. Las modalidades de la innovación, la lógica de servicio y las operaciones de servicio que intervienen en la regulación del acceso a las unidades de cuidados intensivos se describen en los cinco servicios constitutivos identificados. Basándose en el concepto de innovación como las variaciones en los componentes de los vectores de características de los productos de servicio, fué posible identificar la variedad de los procesos innovadores en los diferentes servicios y

\footnotetext{
${ }^{1}$ Submetido em 19 de agosto de 2013. Aceito em 14 de fevereiro de 2014. O artigo foi avaliado segundo o processo de duplo anonimato e avaliado pelo editor. Editores responsáveis: Márcio Augusto Gonçalves e Lucas Maia dos Santos. Reprodução parcial ou total e trabalhos derivativos permitidos com a citação apropriada da fonte.
} 
en organizaciones distintas. El sector público actuó como protagonista del proceso de innovación y como inductor de innovaciones en el sector privado. Las mejoras significativas se encontraron en la equidad en el acceso. Las barreras estructurales y de gestión se mantienen y evitan nuevos avances en la integralidad de la atención sanitaria. Entre las limitaciones locales, se destacan las deficiencias en el sistema de contra-referencia y en la prestación de servicios de salud de mediana complejidad.

PALABRAS-CLAVE: innovación en los servicios, la regulación de acceso, sistema único de salud

\section{Introdução}

$\mathrm{O}$ artigo apresenta os resultados de pesquisa que teve por objetivo descrever o processo de inovação na regulação de acesso ao serviço de internação em unidade de terapia intensiva (UTI) no serviço público de saúde do Distrito Federal (DF). A análise é realizada com fundamento na abordagem integradora da teoria da inovação em serviços (GALLOUJ, 2002), a qual foi mobilizada para caracterizar os diferentes serviços que compõem a regulação, descrever os modos de inovação que se desenvolveram na implementação da regulação e, por fim, examinar os efeitos destas inovações sobre os objetivos declarados da regulação no DF.

Os estudos sobre inovação têm contribuído para a análise da evolução das economias modernas. Porém, ao focar apenas nas inovações geradas nas firmas sob regulação de mercado, este enfoque tem proporcionado respostas parciais. O setor público tem sido tratado como caudatário e periférico nos processos de inovação (KOCH; HAUKNES, 2005; WINDRUM; GARCÍA-GOÑI, 2008). Além disso, as atividades de serviços, privadas ou públicas, são majoritariamente abordadas sob enfoque tecnicista e subordinado (GALLOUJ, 2002). Analisar inovações nos serviços de saúde requer o enfrentamento destes vieses sob uma abordagem integradora (GALLOUJ, 2002).

Do ponto de vista teórico, o setor de saúde é objeto de diversos estudos sobre inovação em serviços, pois nele existem complexidades que permitem compreender melhor as variáveis que atuam sobre os processos de inovação. Do ponto de vista da política de saúde, um dos problemas que ganharam relevância na agenda de discussões sobre o Sistema Único de Saúde brasileiro foi a oferta insuficiente de certos serviços de saúde, em especial os de alta complexidade, com a consequente restrição do acesso ao direito à saúde. A restrição ao acesso é agravada pela falta de priorização do acesso segundo a necessidade de atendimento, com efeito negativo sobre a equidade do sistema (SANTOS et al, 2008).

A regulação assistencial, na qual se insere a regulação de internação em UTI (RIUTI), tem sido apresentada como uma proposta que visa contribuir para a concretização dos princípios de acesso equitativo e atenção integral no Sistema Único de Saúde (SUS) brasileiro, bem como alcançar ganhos de eficiência (CONASS, 2007). Consiste na intermediação da oferta e da procura de serviços públicos de saúde por meio de centrais de regulação. A implantação de controles de acesso aos serviços de saúde caracteriza-se como mecanismo racionalizador, mas sua efetividade está em discussão. Existe, portanto, campo para investigar como se desenvolve o processo de inovação em RIUTI.

A pesquisa desenvolvida abarcou hospitais públicos e privados, com contratos em vigor para uso de leitos de UTI pela Secretaria de Estado de Saúde do DF (SES/DF), bem como o complexo regulatório da SES, na área de RIUTI. O período analisado inicia-se com a introdução da RIUTI no DF, em 2006, até o encerramento da coleta de dados em dezembro de 2010. Os dados foram coletados por meio de pesquisa documental e entrevistas semi-estruturadas e foram objeto de análise de conteúdo (BARDIN, 2009), apoiada pelo software Atlas TI 5.0. 


\section{Regulação assistencial}

A regulação do acesso à assistência também é chamada de regulação de acesso ou de regulação assistencial (BRASIL, 2008), sendo esta última a nomenclatura adotada neste artigo.

De perspectiva bastante geral, a regulação assistencial pode ser entendida como "intervenção de um terceiro entre a demanda do usuário e a prestação efetiva do ato de saúde (...)” (SANTOS; MERHY, 2006, p. 27). O Pacto pela Saúde define regulação assistencial como o "conjunto de relações, saberes, tecnologias e ações que intermedeiam a demanda dos usuários por serviços de saúde e o acesso a eles" (CONASS, 2007, p. 31). Essa definição deixa entrever que o processo de regulação envolve conhecimento técnico tanto em administração quanto no serviço de saúde prestado (SANTOS et al, 2008).

A operacionalização da regulação é estabelecida pelo complexo regulador e suas unidades operacionais. Abrange a regulação médica, exercendo autoridade sanitária para a garantia do acesso, baseada em protocolos, classificação de risco e demais critérios de priorização (BRASIL, 2008, art. $2^{\circ}$, III). Os complexos reguladores são formados por unidades operacionais denominadas centrais de regulação (BRASIL, 2008, art. $7^{\circ}$ ).

$\mathrm{Na}$ modelagem do sistema, os complexos reguladores contariam com uma série de instrumentos para operacionalizar a intermediação. Com relação à oferta, os principais instrumentos são o Cadastro Nacional de Estabelecimentos de Saúde (CNES), a Programação Pactuada e Integrada (PPI) e o Programa Nacional de Avaliação de Serviços de Saúde (PNASS). Com relação à demanda, o principal instrumento seria o Cartão Nacional de Saúde.

Todo o aparato de sistematização de informações, de estabelecimento de fluxos assistenciais, pactuação intergestores de referências, planejamento da oferta e controle de qualidade colocam a regulação assistencial no centro de um movimento maior de reorganização do sistema de saúde. A efetivação da regulação assistencial depende do adequado funcionamento desse arsenal complexo de instrumentos de gestão. Contudo, foge ao escopo desta pesquisa o exame de sua implementação.

Para Magalhães (2002 apud SANTOS; MERHY, 2006), em um sistema regulado, o usuário, ao adentrar a rede de serviços, passa a ser direcionado pelo sistema. Assim, na regulação do acesso por meio das centrais de regulação, restringe-se o direito de escolha do usuário e em troca lhe é oferecida a promessa de garantia de acesso. No entanto, para alguns gestores, corre-se o risco de que a regulação assistencial transforme-se em apenas mais um mecanismo de racionalização do uso de recursos disponíveis no lugar de contribuir para efetivar os princípios da integralidade e da equidade (IBANHES et al, 2007).

\section{Regulação assistencial no Distrito Federal}

O sistema de saúde do DF encontra-se em contexto peculiar. O DF tem atribuições e competências tributárias de estado e de município simultaneamente (BRASIL, 2004a), o que aumenta suas responsabilidades na prestação de serviço, mas igualmente aumenta seu poder de arrecadação. O DF foi criado em 1960 e, em 2009, contava com população estimada de 2,6 milhões habitantes (IBGE, 2009).

Do lado da receita, o DF ainda recebe assistência financeira da União para os serviços de saúde (BRASIL, 2004a, art. 21; BRASIL, 2002b). Do lado da demanda, o sistema de saúde do DF atende também não moradores, principalmente provenientes dos 22 municípios médios e pequenos dos estados de Goiás e Minas Gerais, que formam a Região Integrada de Desenvolvimento do Distrito Federal e Entorno (RIDE). Esse fluxo adicional de usuários corresponde a cerca de 15,6\% (internação hospitalar) a 19,6\% (ambulatório e emergência) dos 
atendimentos no sistema de saúde local (PIRES et al, 2010). Deve-se levar em conta também que $21,9 \%$ da população do DF é coberta por planos de saúde suplementar (ANS, 2009).

No DF há 341 leitos de UTI disponíveis para o SUS, sendo $62 \%$ na rede própria. Cerca de $92 \%$ dos leitos de UTI disponíveis para o SUS encontram-se regulados. O Ministério da Saúde (BRASIL, 2002c) estima a necessidade de que $4 \%$ a $10 \%$ do total de leitos hospitalares sejam de UTI. Por sua vez, a necessidade de leitos hospitalares seria de 2,5 a 3 leitos por 1.000 habitantes. Assim, considerando a necessidade mínima de leitos hospitalares para 2,6 milhões de habitantes, o número de leitos de UTI disponíveis para o SUS no DF seria o equivalente a 5,2\% dos leitos hospitalares, o que atende ao parâmetro do Ministério.

Os primeiros movimentos em direção à implantação da regulação assistencial no DF de forma sistematizada começaram em 2004, com a criação do Grupo Técnico de Regulação Assistencial (GTRA) (DF, 2004). O grupo revisou contratos e convênios, definiu fluxos e contrafluxos e estimou demanda reprimida por especialidade.

Em agosto de 2006, após diversas iniciativas de regulação na área ambulatorial, foi criada a Central de Regulação de Internação Hospitalar (CRIH) (DF, 2006b). A CRIH é o principal operador do sistema de RIUTI, constituindo-se em unidade organizacional da administração direta, que atua de forma alinhada com os objetivos governamentais, sob controle hierárquico. Na ocasião, foram incluídos na regulação os leitos de terapia intensiva neonatal, pediátrica e adulto dos hospitais da rede própria, conveniada e contratada do DF. Foi determinado que caberia ao SAMU o transporte inter-hospitalar de pacientes que necessitassem de internação em UTI. Ato contínuo, a SES publicou diretrizes para admissão e alta em terapia intensiva, o que caracterizou a aprovação do primeiro protocolo clínico e de regulação desta área no DF (DF, 2006a).

O complexo regulador do DF é composto pelas centrais de Regulação da Internação Hospitalar (CRIH), de Marcação de Consultas e Exames (CMCE) e pela Central Estadual de Regulação da Alta Complexidade (CERAC) (DF, 2009).

A ferramenta de apoio informatizado para gerenciar a regulação ambulatorial no DF é o Sisreg. Na RIUTI é usado módulo de sistema WEB desenvolvido por empresa prestadora de serviços de informática para a SES/DF, chamado TrakCareTM. Abrange ainda o módulo de regulação de medicamentos e o prontuário eletrônico, este implantado em 5 dos 15 hospitais distritais.

O processo está desenhado para funcionar por meio do software, mas, na prática, há contatos telefônicos frequentes para completar informações do laudo técnico, informar a unidade executante sobre o encaminhamento do paciente e a unidade solicitante sobre a autorização da internação.

Apesar de o Manual de Implantação de Complexos Reguladores (BRASIL, 2006b) orientar que o escopo da CRIH deve ser os leitos das diversas clínicas, de UTI e de retaguarda aos prontos-socorros, o DF, demais unidades da federação e municípios têm autonomia para organizar seus complexos reguladores. O DF, no período analisado, era o único cuja CRIH regulava apenas leitos de UTI.

\section{Marco teórico}

O marco teórico deste artigo é a teoria da inovação em serviços em sua abordagem integradora (GALLOUJ, 2002). Tal enfoque, apesar de não ser específico para o setor com regulação política, vem sendo usada para estudar o setor público, principalmente os serviços de saúde em hospitais (WINDRUM; GARCÍA-GOÑI, 2008). Estudos sobre inovação m serviços tem contribuído decisivamente para a melhor compreensão da inovação no setor público (LIMA; VARGAS, 2012). Complementarmente, deve-se destacar que a teoria de inovação no 
setor público, com fundamento em escolas de administração pública, vem se fortalecendo a partir de estudos financiados pela União Europeia. Neste contexto, Koch e Hauknes (2005, p. 9) apresentam uma definição de inovação no setor público que reconhece o caráter contextual da natureza do resultado da inovação: "Inovação é a implementação ou desempenho de uma nova forma específica ou repertório de ação social, implementada deliberadamente por uma entidade no contexto dos objetivos e funcionalidades de suas atividades".

Essa definição permite caracterizar a regulação assistencial como uma inovação no setor público e justificar o uso da teoria da inovação em serviços para analisá-la. A RIUTI representa a centralização da intermediação entre oferta e procura pelo serviço de internação em UTI, configurando uma nova forma de implementação desse serviço de saúde. Além disso, foi implantada deliberadamente pela SES/DF por meio de normas, tendo em vista o princípio da legalidade que impera no setor público. Essa nova forma de prestar o serviço foi implantada no contexto local do sistema de saúde, que apresenta deficiências comuns ao SUS, mas com especificidades decorrentes da estrutura local, da pressão de demanda proveniente de outros entes federados e pelas condições de financiamento.

Essa interpretação é coerente com a teoria evolucionária da mudança econômica (NELSON; WINTER, 1982), que é uma das fontes da abordagem integradora da teoria da inovação em serviços. Nesta tradição, Gallouj (2002, p. 132) afirma que a inovação não é um resultado final e conclusivo (artefato), mas um processo de resolução de problemas, no qual a aprendizagem e interações múltiplas entre os agentes têm o papel principal. Segundo a teoria evolucionária, a inovação seria um processo de solução de problemas, dentro de um paradigma, o qual induziria soluções cumulativas, formando trajetórias de soluções (path dependence), o que pode até cercear a capacidade de encontrar soluções fora dessas trajetórias (lock-in) (SBICCA; FERNANDES, 2005).

A abordagem integradora da inovação em serviços ambiciona juntar bens e serviços em uma mesma teoria da inovação (GALLOUJ, 2002). Uma das justificativas para o desenvolvimento dessa abordagem é a existência de especificidades no produto dos serviços e nos processos de inovação neste setor que fazem com que a teoria da inovação construída com foco na indústria seja insuficiente para ajudar a compreendê-los. Sendo assim, é necessário desenvolver uma nova abordagem, suficientemente flexível e abrangente que permita examinar o processo de inovação nesses dois setores. Uma forma de interpretar a abordagem integradora é por meio da discussão das especificidades dos serviços e das formulações que foram desenvolvidas para tratá-las. Três modelos analíticos destacam-se na abordagem integradora da teoria da inovação em serviços, que tratam explicitamente de especificidades dos serviços: o da relação de serviço (GADREY, 2001), o da decomposição funcional do produto (GALLOUJ, 2002) e o da representação vetorial do produto (GALLOUJ; WEINSTEIN, 1997). Este artigo limita-se a expor este último que será mobilizado para análise dos dados coletados.

Gallouj e Weinstein (1997) estenderam e aperfeiçoaram um modelo anterior de cunho tecnicista (SAVIOTTI; METCALFE, 1984), com base em seus estudos sobre inovação em serviços. Assim, no modelo de Gallouj e Weinstein (1997), o produto do serviço é representado pelos vetores $[\mathrm{C}]$, de competências dos prestadores, $[\mathrm{T}]$ de competências técnicas tangíveis e intangíveis, pelo vetor [C'] de competências dos clientes e pelo vetor [Y] de características finais do produto, percebidas diretamente pelo cliente. 
Figura 1 - Representação geral do produto em termos de vetores de características

Fonte: Adaptado de Gallouj, 2002, p.58

Essa formulação permite abranger duas das especificidades do setor: a diversidade e o componente relacional dos serviços. O tratamento analítico dessa última ocorre pela incorporação de um vetor de competências dos clientes [C'] ao modelo. Como essa formulação é uma generalização do modelo de Saviotti e Metcalfe (1984) para bens, ela permite analisar de forma unificada tanto bens quanto serviços. Outro avanço teórico dessa é a incorporação à análise das características técnicas imateriais, que são sistemas de competências formalizadas e codificadas (GALLOUJ, 2002, p. 56). O desenvolvimento do modelo sob uma perspectiva evolucionária permite definir a inovação em termos de variações nos componentes dos vetores de características. Essas variações definem seis modos segundo os quais ocorre o processo de inovação (GALLOUJ, 2002).

O primeiro modo ou modelo é o de inovação radical, no qual ocorre a criação de um novo conjunto de vetores de características, configurando um novo produto. Segundo o autor, a definição mais ampla deste modelo abrange ainda o caso no qual surgem novas competências de clientes e produtores, bem como novas características técnicas materiais e imateriais, mas as características finais do produto não se modificam significativamente.

A inovação de melhoria envolve mudança na qualidade das características do produto [Y], por meio de aperfeiçoamentos de componentes do vetor de competências diretas dos prestadores ou de características técnicas. A inovação incremental descreve a ocorrência de aperfeiçoamento das características finais do produto por meio da adição ou substituição de componentes dos vetores por novas características. A inovação por recombinação é o modelo segundo o qual componentes dos vetores de características dos produtos dos serviços são combinados ou dissociados para formar novo produto. A inovação por formalização é a sistematização documentada das características do produto, tornando-as mais visíveis e padronizadas. A inovação ad hoc é a construção conjunta de uma solução para o problema colocado pelo cliente, por meio da interação entre este e o produtor/prestador de serviço, o que provoca mudanças nas características do serviço. 


\section{Método}

Esta pesquisa está registrada sob o número 117/10 no Comitê de Ética em Pesquisa da Faculdade de Ciências da Saúde da UnB, tendo sido aprovada pelo Parecer Complementar CEP/FS-UnB No 117/10, de 5/10/2010.

A pesquisa realizada é qualitativa e descritiva. A estratégia metodológica usada foi o estudo de caso único integrado (YIN, 2010). Trata-se de desenho no qual existe um caso único (a RIUTI), mas há múltiplas unidades de análise (os serviços elementares constitutivos). O desenho da pesquisa inclui a intenção de analisar o caso em relação às condições contextuais, pois os limites entre os casos integrados e entre os casos e o contexto não são rígidos.

Esse desenho é consistente com a teoria de inovação em serviços, que reconhece a diversidade dos serviços e procura tratar o fenômeno da inovação em detalhes. Essa abordagem evita analisar o fenômeno em nível excessivamente abstrato, expondo de forma mais clara os dados de aspectos específicos (YIN, 2010, p. 69 e 73-76).

Foram realizadas entrevistas com 35 gestores/especialistas da SES/DF, bem como profissionais médicos e não médicos. As entrevistas totalizaram $15 \mathrm{~h}$ e $17 \mathrm{~min}$ de áudio e foram transcritas, resultando em 300 páginas de texto analisadas por meio de análise de conteúdo (BARDIN, 2009), assistida pelo software Atlas.TI 5.0.

A análise identificou 618 menções, assinaladas por 38 códigos, associados a nove variáveis: 1) origens da RIUTI, 2) mudanças nas competências diretas dos prestadores [C], 3) desenvolvimento de novas formas de relacionamento entre prestadores e usuários, 4) Mudanças nas competências técnicas [T], 5) Mudanças nas competências dos clientes [C'], 6) Resultados do processo de inovação em RIUTI, 7) Propulsores da implantação da RIUTI, 8) Obstáculos da implantação da RIUTI, 9) Mudanças nas características finais do serviço [Y].

A regra de enumeração das unidades de registro foi a da presença (ou ausência). Dúvidas sobre a codificação de temas ou mesmo sobre sua categorização foram dirimidas pela observação do contexto no qual a fala se inseria e da ênfase aplicada pelo entrevistado.

Para operacionalizar um plano de análise que abrangesse a multiplicidade de funções observadas e que pudesse ser combinado com a representação vetorial do serviço (ver figura 1), foi usada a noção de que o serviço pode ser decomposto em diferentes serviços elementares (Sij), onde i representa o serviço e j representa o estabelecimento onde o serviço i é prestado, quando há mais de um estabelecimento que o presta (DJELLAL; GALLOUJ, 2005).

Sinteticamente, os serviços elementares são descritos a seguir. A solicitação de vaga (S1) é realizada no hospital solicitante pelo médico solicitante que assiste o paciente. $\mathrm{O}$ processo regulatório de internação em UTI inicia-se com a inserção no TrakCare da solicitação de internação pela unidade solicitante. A intermediação de vaga (S3) é realizada pela central de regulação, o que abrange a priorização do paciente segundo o risco (DF, 2006a). Simultaneamente, o enfermeiro controlador busca a vaga no sistema. Encontrada a vaga, o médico regulador identifica o médico que receberá o paciente e lhe repassa informações sobre as condições do paciente. O médico regulador comunica a alocação de vaga para a unidade solicitante.

O atendimento ao paciente (S2) abrange a preparação do leito, a liberação da vaga no sistema de regulação e os cuidados médicos e de enfermagem ao paciente recebido. A auditoria de contas (S4) é serviço administrativo prestado pela Central de Regulação à SES/DF, que precisa ter garantia de que os valores apresentados pelos hospitais contratados são efetivamente devidos. A supervisão médica (S5) é prestada pela Central de Regulação, por médicos supervisores, para a SES/DF, que precisa ter garantia de que os procedimento realizados nos pacientes encaminhados para os hospitais privados são corretos e justificam a despesa incorrida. 
Os dados foram coletados em seis estabelecimentos de saúde, três públicos e três privados, sendo um público sem UTI. O serviço $\mathrm{S} 1$ foi analisado neste último. O Serviço S2 é desenvolvido em sete UTIs em cinco estabelecimentos de saúde. Cada um desses serviços foi considerado uma unidade integrada de análise do caso único RIUTI.

O modelo conceitual da pesquisa é a representação do serviço como conjunto de vetores de características (Figura 1). Dessa forma, cada um dos serviços elementares constituintes (DJELLAL; GALLOUJ, 2005) da RIUTI (Sij) pode ser entendido por meio de um conjunto próprio de vetores de características.

\section{Resultados e discussão}

Nesta seção os resultados serão apresentados e discutidos à luz da abordagem integradora da teoria da inovação em serviços, considerando o estudo de caso único integrado, representado pela RIUTI no DF. A discussão terá por base os resultados sintetizados nos Quadros 1 e 2.

\section{Quadro 1 - Mudanças nos vetores de características da RIUTI, por serviço elementar constitutivo e estabelecimento}

\begin{tabular}{|c|c|c|c|c|c|c|c|c|c|c|c|}
\hline Mudanças vetoriais / Serviços elementares & $\mathrm{S} 1$ & S21 & S22 & S23 & S24 & S25 & S26 & S27 & S3 & S4 & S5 \\
\hline Uso do TrakCare $[\mathrm{T}]$ & $\mathrm{X}$ & $\mathrm{X}$ & $\mathrm{X}$ & $\mathrm{X}$ & $\mathrm{X}$ & $\mathrm{X}$ & $\mathrm{X}$ & $\mathrm{X}$ & $X$ & & $X$ \\
\hline $\begin{array}{l}\text { Sistematização documentada de procedimentos e } \\
\text { critérios }[\mathrm{T}]\end{array}$ & $\mathrm{X}$ & $\mathrm{X}$ & $\mathrm{X}$ & $\mathrm{X}$ & $\mathrm{X}$ & $\mathrm{X}$ & $\mathrm{X}$ & $\mathrm{X}$ & $\mathrm{X}$ & & $X$ \\
\hline Inclusão de novos equipamentos [T] & & & & & & & $\mathrm{X}$ & & & & \\
\hline $\begin{array}{l}\text { Desenvolvimento de competência na operação de } \\
\text { sistema informatizado [C] }\end{array}$ & $\mathrm{X}$ & $\mathrm{X}$ & & $\mathrm{X}$ & $\mathrm{X}$ & & & $\mathrm{X}$ & & & \\
\hline $\begin{array}{l}\text { Desenvolvimento de competências profissionais } \\
\text { [C] }\end{array}$ & & & $\mathrm{X}$ & & & & $\mathrm{X}$ & $\mathrm{X}$ & & & \\
\hline Aumento de pessoal e de produção do serviço [C] & & & & & $\mathrm{X}$ & $\mathrm{X}$ & $\mathrm{X}$ & $\mathrm{X}$ & & $\mathrm{X}$ & \\
\hline Criação de novas funções/ serviços [C] & & & & & & & & & $\mathrm{X}$ & $\mathrm{X}$ & $\mathrm{X}$ \\
\hline Mudança na organização do serviço [C] & & & & & & & & & & $\mathrm{X}$ & \\
\hline $\begin{array}{l}\text { Maior variedade de situações clínicas dos } \\
\text { pacientes [C'] }\end{array}$ & & & $\mathrm{X}$ & $\mathrm{X}$ & & & & $\mathrm{X}$ & & & \\
\hline $\begin{array}{l}\text { Maior gravidade da situação clínica dos pacientes } \\
\text { [C'] }\end{array}$ & & & & & & $\mathrm{X}$ & $\mathrm{X}$ & & & & \\
\hline
\end{tabular}

\section{Fonte: elaborado pelos autores.}

Sintetizando os resultados da pesquisa, o Quadro 1 apresenta as principais mudanças nos vetores de características dos serviços elementares constitutivos (DJELLAL; GALLOUJ, 2005) da RIUTI. Considerando a definição de Gallouj (2002) da inovação como variações nos componentes desses vetores, foi possível elaborar o Quadro 2, que detalha os diferentes modos de inovação que ocorrem no processo de inovação em RIUTI.

Com base na análise das variações nos componentes dos vetores de características dos produtos dos serviços elementares (Quadro 1), é possível identificar que houve a criação de novas funções e serviços no processo de inovação em RIUTI (S3, S4, S5), o que é identificado com o modo radical de inovação (Quadro 2). Esse é o modo mais significativo de inovação, pois representa a criação de novos serviços no sistema de saúde do DF. 


\section{Quadro 2 - Formas de inovação na RIUTI, por serviço elementar constitutivo e estabelecimento}

\begin{tabular}{|l|c|c|c|c|c|}
\hline $\begin{array}{l}\text { Serviços } \\
\text { elementares }\end{array}$ & Incremental & Formalização & Melhoria & Radical & De mercado \\
\hline S1 & $\mathrm{X}$ & $\mathrm{X}$ & & & \\
\hline S21 & $\mathrm{X}$ & $\mathrm{X}$ & & & \\
\hline S22 & $\mathrm{X}$ & $\mathrm{X}$ & $\mathrm{X}$ & & \\
\hline S23 & $\mathrm{X}$ & $\mathrm{X}$ & $\mathrm{X}$ & & \\
\hline S24 & $\mathrm{X}$ & $\mathrm{X}$ & $\mathrm{X}$ & & $\mathrm{X}$ \\
\hline S25 & $\mathrm{X}$ & $\mathrm{X}$ & $\mathrm{X}$ & & $\mathrm{X}$ \\
\hline S26 & $\mathrm{X}$ & $\mathrm{X}$ & $\mathrm{X}$ & & $\mathrm{X}$ \\
\hline S27 & $\mathrm{X}$ & $\mathrm{X}$ & & $\mathrm{X}$ & \\
\hline S3 & & & $\mathrm{X}$ & $\mathrm{X}$ & \\
\hline S4 & $\mathrm{X}$ & $\mathrm{X}$ & & $\mathrm{X}$ & \\
\hline S5 & & & & \\
\hline
\end{tabular}

Fonte: elaboração do autor, com base na análise de conteúdo das entrevistas.

Como consequência e para viabilizar a RIUTI, surgiram processos de inovação incremental, por formalização e de melhoria. As inovações incrementais são entendidas como adição ou substituição de componentes dos vetores por novas características. As por formalização identificam-se com a sistematização documentada das características do produto, tornando-as mais visíveis e padronizadas. Por fim, inovações de melhoria envolvem mudanças na qualidade dos componentes dos vetores de características dos produtos (GALLOUJ, 2002).

A análise do Quadro 1 revela que a formalização de procedimentos e critérios e o acréscimo de novas competências e características foram modos disseminados de inovação nos serviços constitutivos da RIUTI.

Para viabilizar a implantação da RIUTI, foi necessário sistematizar e documentar os procedimentos a serem seguidos pelos prestadores de serviço e os critérios de priorização da internação em UTI, conforme a necessidade dos pacientes. Esse processo gerou a publicação de portarias que normatizam a RIUTI, caracterizando a inovação por formalização.

Além disso, o processo de inovação na RIUTI provou estar muito relacionado a adições de novos componentes nos vetores de competências diretas dos prestadores e de características técnicas. Para viabilizar a regulação, foi necessário incorporar o sistema eletrônico de regulação ao vetor de características técnicas e que os prestadores desenvolvessem competência para operá-lo. Esses processos caracterizam inovações incrementais.

As duas inovações mencionadas abrangeram todos os serviços elementares, independentemente do tipo de estabelecimento prestador se serviço, exceto a auditoria de contas (S4), que não usa o sistema de regulação. Era esperado que informatização e normatização se apresentassem em conjunto, pois a construção do fluxo do sistema eletrônico baseou-se nas normas procedimentais definidas pela SES/DF.

As situações aqui descritas permitem entender a RIUTI como um processo de centralização do serviço de intermediação de vagas, viabilizado pela informatização dos serviços elementares, pela normatização de procedimentos e pela criação dos serviços de auditoria de contas e de supervisão médica.

Além disso, a implantação da RIUTI ocorreu em contexto de reorganização do sistema de assistência em emergência. Assim, foram contratados e regulados leitos de UTI em hospitais privados e houve o redirecionamento de pacientes para UTI públicas que antes prestavam um serviço mais especializado. 
Nos estabelecimentos privados, a contratação dos leitos representou uma inovação de mercado (SCHUMPETER, 1982), pois abriu o SUS ao serviço de atendimento ao paciente em UTI (S24, S25, S26 e S27). Nos mencionados serviços, a abertura de mercado exigiu uma série de inovações por formalização, incrementais e de melhoria. Respectivamente, essas inovações nos serviços responderam à necessidade de seguir novos procedimentos em vigor na RIUTI, de usar novos equipamentos e software, adquirir novas habilidades, bem como de aumentar pessoal e produção para atender a nova demanda. No setor privado, a contratação com a SES/DF, decorrente da reorganização do sistema, da qual a RIUTI faz parte, foi um fator de sustentação de estratégias empresariais de expansão de serviços.

Os efeitos da inovação em RIUTI sobre os estabelecimentos privados ilumina outra face do processo de inovação na economia, na qual o setor público atua como protagonista, induzindo inovação no setor privado (KOCH; HAUKNES, 2005). Essa é a situação contrária à destacada na literatura de inovação de abordagem tecnicista (GALLOUJ, 2002), na qual a indústria de transformação é protagonista e o setor público é coadjuvante.

Outra situação de interesse teórico corresponde à mudança na variedade e gravidade dos casos clínicos recebidos por estabelecimentos privados e por estabelecimentos públicos, anteriormente especializados. Esse evento provocou processos localizados de inovação de melhoria, representados pelo aperfeiçoamento de competências profissionais dos prestadores do serviço de atendimento ao paciente (S22, S26 e S27), e incremental, representado pela incorporação de novos equipamentos (S26).

Trata-se de caso particular de interações entre o vetor de competências dos clientes/usuários [C'] com os vetores de competências diretas dos prestadores [C] e de características técnicas [T]. Onde as variações em [C'] não encontraram características em [C] e [T] que respondiam às novas necessidades do serviço, houve o aperfeiçoamento de competências diretas dos prestadores e a incorporação de equipamentos. Apresenta-se aqui um curioso processo local de inovação, mobilizado pelas competências dos usuários, mas com relação de serviço fraca (GADREY, 2001). Diferentemente, a literatura associa a inovação decorrente da interação entre $[\mathrm{C}]$ e [C'] à relação de serviço intensa e ao modo de inovação ad hoc, caracterizado pelo processo de coprodução de inovações (GALLOUJ, 2002).

Houve grande variedade de mudanças nos vetores de características dos produtos dos serviços. Apenas dois pares de serviços se formaram pela identidade na mudança dos componentes de seus vetores: S3 e S5, S1 e S21. Todos os outros serviços elementares apresentaram particularidades que os distinguiram dos demais. Apesar dessa grande variedade de modos de inovação presente na implantação da RIUTI, observa-se a ausência de inovações ad hoc e por recombinação. Possíveis razões para esses fatos são, respectivamente, o ambiente de baixa interação verbal entre prestadores e pacientes e a importância da criação de novos serviços no DF para viabilizar a RIUTI, no lugar de combinar características de serviços préexistentes como conceituado na inovação de formalização.

O exame dos documentos distritais revela a expectativa de melhoria nas dimensões de eficiência, equidade e integralidade da assistência. Segundo os entrevistados, é possível argumentar que o processo de inovação em RIUTI, em interação com o contexto do sistema de saúde do DF, provocou efeitos em cada uma das três dimensões identificadas, nem sempre no sentido de aperfeiçoamento, conforme retratado na Figura 2. 


\section{Figura 2 - O processo de inovação em RIUTI no DF}

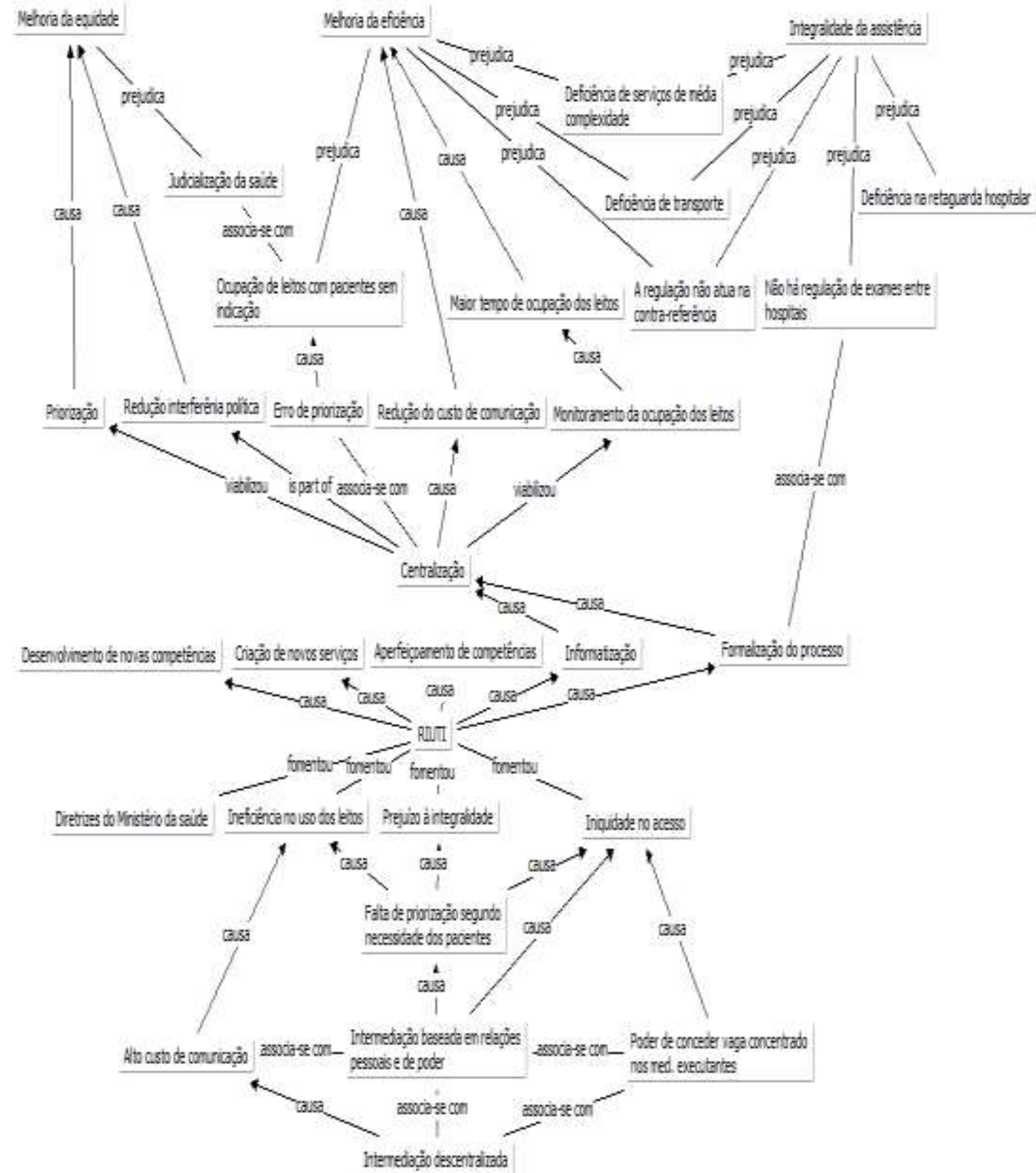

Fonte: elaboração dos autores.

A figura sintetiza a interpretação sobre o entendimento dos entrevistados acerca do processo de inovação em RIUTI. A RIUTI surgiu como um processo de resolução de problemas (GALLOUJ, 2002), fomentado por diretrizes do SUS no sentido de incentivar a implantação da regulação assistencial no Brasil, inclusive com concessão de incentivos financeiros (NASCIMENTO et al, 2009).

Assim, na Figura 2, a parte inferior, até o ponto de convergência na RIUTI representa as motivações que estiveram na origem do processo de inovação analisado. São problemas e 
fatores institucionais que não eram exclusivos do DF, mas tinham suas peculiaridades locais, como, por exemplo, a intensidade do déficit de oferta de serviços em UTI. A própria solução local para a regulação foi idiossincrática em diferentes aspectos, como a regulação apenas das internações em UTI e a contratação de software próprio de regulação.

A partir da base da figura 2, observa-se que, antes da implantação da regulação, a intermediação entre a procura e a oferta por vagas de internação em UTI era descentralizada. Os médicos e enfermeiros dos estabelecimentos solicitantes incorriam em alto custo de comunicação ao ligar para os estabelecimentos com UTI e explicar o caso até conseguirem vaga. Nesse processo, podiam ser submetidos a situações de incerteza pela falta de documentação do procedimento seguido e estarem sujeitos a constrangimentos causados pela necessidade de recorrer a relações pessoais. Os médicos executantes detinham o poder de conceder a vaga solicitada, o que lhes trazia prestígio, mas, em contrapartida, também estavam sujeitos a situações nas quais relações de poder lhes impunham certas ações.

Essa situação impedia que as demandas, também descentralizadas, fossem priorizadas segundo a necessidade dos pacientes por internação. O processo trazia prejuízos aos princípios doutrinários do SUS de equidade de acesso e integralidade da assistência, bem como ao princípio da eficiência na Administração Pública.

As principais inovações em decorrência da implantação da RIUTI estão representadas na parte intermediária da Figura 2: a formalização de todo o processo, em especial dos critérios de priorização de pacientes segundo a gravidade do quadro clínico, a informatização e centralização da intermediação, o que viabilizou o monitoramento em tempo real dos leitos de UTI. Paralelamente, houve processos generalizados e localizados de desenvolvimento de novas competências e de aperfeiçoamento das existentes. Em alguns serviços contratados, houve expansão da produção, do quadro de pessoal e a incorporação de equipamentos. Foram criados os serviços de supervisão médica, de auditoria de contas e a de intermediação centralizada de vagas. A teoria da inovação em serviços identifica essas mudanças nas características dos diferentes serviços constitutivos da RIUTI como inovações radicais, incrementais, de melhoria e de formalização (GALLOUJ, 2002).

Na parte superior da Figura 2 estão representados os efeitos esperados e não esperados, desejados e indesejados da RIUTI. Os efeitos esperados e desejados pela SES/DF ao implantar a regulação foram construídos no processo de diagnóstico e discussão de soluções, desenvolvido nacionalmente no âmbito do SUS, e apropriados pela administração local, conforme explicitado nos normativos da regulação. Aliás, a própria RIUTI foi um novo serviço que surgiu no processo de interação entre os atores do SUS. Na teoria de inovação em serviços (GADREY, 2001), esses efeitos são identificados com os resultados da inovação, isto é, os resultados produzidos pelos produtos dos serviços, sejam eles novos ou modificados em suas características. A construção da RIUTI e de seus efeitos esperados, explicitada nos diferentes fóruns de discussão colegiada do SUS, acrescentam evidência ao caráter convencional do produto dos serviços (GADREY, 2001).

A inovação no serviço produziu efeitos no sentido de reduzir os problemas que a motivaram. A eficiência foi melhorada pela redução do custo de comunicação para a intermediação de vagas e pelo monitoramento dos leitos, o que permitiu aumentar o tempo de seu uso. No entanto, problemas estruturais e de organização do sistema de saúde do DF atuam no sentido oposto. A deficiência dos meios de transporte contribui para a demora excessiva na recepção dos pacientes nas UTI. A regulação não atua sobre a contrarreferência, o que aliado à deficiência nos serviços de média complexidade, contribui para que pacientes sem indicação permaneçam ocupando leitos de UTI.

A RIUTI expôs problemas que prejudicam a integralidade da assistência à saúde. Não existe regulação de exames para pacientes de UTI entre hospitais da rede, o que aliado à deficiência na retaguarda hospitalar para a UTI e à deficiência de transporte, faz com que alguns 
pacientes deixem de realizar exames e procedimentos indicados. Além disso, pacientes sem indicação de permanecer na UTI podem ter o tratamento interrompido por falta de vaga em serviços de menor complexidade.

Houve melhoria expressiva na equidade do acesso à internação em UTI pela supressão das influências pessoais e políticas na obtenção de vagas e pela aplicação de critérios de priorização de pacientes segundo a gravidade de seu quadro clínico. A judicialização do acesso à internação em UTI por meio da expedição de mandados judiciais interfere na alocação de vagas por critério de necessidade. Contudo, pode também ser interpretada como reação à deficiência no número de vagas e como ação a favor da integralidade da assistência.

Dessa forma, o modelo representado na Figura 2 faz uma conexão entre a análise do processo de inovação na RIUTI no DF e a política de saúde local, representada por fatores que estavam na origem ou motivação da implantação da RIUTI e posteriormente nos seus resultados para o sistema de saúde.

\section{Considerações Finais}

O objetivo do artigo foi descrever os processos de inovação desenvolvidos ao longo da implantação da RIUTI no DF nos serviços que a constituem. Para alcançá-lo, foram descritos os processos de inovação nos diferentes serviços constitutivos da RIUTI. Assim, foi possível identificar inovações nos serviços elementares que compõem a RIUTI, bem como seus efeitos sobre esse serviço de assistência à saúde.

A descrição dos processos de inovação revelou que a implantação da RIUTI demandou o desenvolvimento e o aperfeiçoamento de competências diretas dos diferentes prestadores, a incorporação de características técnicas e a criação de novas funções e serviços. Essas inovações ocorreram de forma bem diversificada em diferentes estabelecimentos e serviços constitutivos.

Igualmente diversificados foram os resultados do processo de inovação sobre os objetivos do SUS de prestar assistência à saúde de forma equânime, integral e eficiente. Fatores de contexto referentes à estrutura e à organização do sistema de saúde do DF atuaram de forma a favorecer ou limitar esses resultados. Ao lado de contribuições significativas para a melhoria da equidade de acesso e da eficiência no uso dos recursos existentes, ficaram mais evidentes as restrições à assistência integral à saúde dos pacientes, em razão da falta de garantia da contrarreferência e da deficiência da oferta de serviços de média complexidade.

O estudo de caso da RIUTI acrescenta evidências sobre o papel de variações no vetor de competências dos usuários sobre o processo de inovação, especialmente sobre o aperfeiçoamento de competências diretas dos prestadores e a incorporação de novas características técnicas ao produto do serviço. Evidencia também situações nas quais o papel protagonista do setor público no processo de inovação induz inovações no setor privado da economia, gerando crescimento, incorporação de competências e de artefatos tecnológicos.

Para os gestores, esta pesquisa oferece uma descrição da percepção dos agentes envolvidos na regulação, tanto nos serviços de atendimento direto ao cidadão quanto nos serviços de suporte, nas esferas privada e pública. Os gestores podem usar esta pesquisa como subsídio ao processo de tomada de decisão sobre quais providências são urgentes e poderão causar maior impacto sobre a qualidade da prestação de serviço. Claramente, a deficiência no sistema de contrarreferência impede avanços na integralidade da assistência, como alertado por Giovanella et al (2002). Há indicações de que a solução ou redução do problema passa pelo aprofundamento do processo de regulação para os demais procedimentos ambulatoriais e hospitalares, pelo aperfeiçoamento do sistema de transporte, pela expansão da produção e da estrutura dos serviços de média complexidade. 
A pesquisa suscitou questões que podem ser objeto de investigação em outros estudos. A RIUTI está inserida na regulação assistencial, que no DF vem sendo implantada se forma extremamente gradual. Apenas consultas e exames identificados como os que apresentam maior deficiência no atendimento foram regulados de início. As internações fora de UTI não foram reguladas nem o agendamento de cirurgias eletivas. A incorporação de novos procedimentos depende do interesse dos coordenadores de área e de uma abordagem apenas consultiva da equipe da Central de Regulação. Não estão claros quais os fatores que modulam a velocidade e a profundidade do processo de inovação em regulação assistencial no DF se financeiros, se políticos, se de capacidade de gestão. Seria interessante investigar em que medida esses fatores atuam como obstáculos ou como propulsores ao processo (KOCH; HAUKNES, 2005; DJELLAL; GALLOUJ, 2008).

Finalmente, cabe destacar que pesquisas que incorporem referencial teórico que abranja as relações de poder no setor público poderiam enriquecer a compreensão do fenômeno da inovação no setor público e possivelmente contribuir para a formulação de teorias localizadas sobre inovação, a exemplo dos estudos de Koch e Hauknes (2005) e de Windrum e García-Goñi (2008).

\section{Referências}

AGÊNCIA NACIONAL DE SAÚDE SUPLEMENTAR (ANS). Caderno de Informação da Saúde Suplementar: beneficiários, operadoras e planos. Rio de Janeiro: ANS, dez. 2009.

BARDIN, L. Análise de Conteúdo. 4. ed. rev. e atual. Lisboa: Edições 70, 2009.

BRASIL. Lei $\mathbf{n}^{\mathbf{0}} \mathbf{1 0 . 6 3 3}$, de 27 de dezembro de 2002. Institui o Fundo Constitucional do Distrito Federal. Brasília, 2002b. Disponível em: <http://www.planalto.gov.br>. Acesso em: 21 out. 2010.

Ministério da Saúde. Portaria no 1.101, de 12 de junho de 2002. Estabelece parâmetros de cobertura assistencial no SUS. Brasília, 2002c. Disponível em: < pnass.datasus.gov.br>. Acesso em: 15 abr. 2010.

Constituição da República Federativa do Brasil, de 1988. Brasília, DF: Câmara dos Deputados, 2004a.

Ministério da Saúde. Manual de Implantação de Complexos Reguladores. Brasília: Ministério da Saúde, 2006b.

Ministério da Saúde. Portaria GM/MS no 1.559, de $1^{\text {o }}$ de agosto de 2008. Institui a Política Nacional de Regulação do Sistema Único de Saúde. Brasília, 2008. Disponível em: <http://bvsms.saude.gov.br>. Acesso em: 22 fev. 2010.

CAMARGOS, C.N.; PRADO, C.C.L.; ASSIS, W.F.; MERÇON, J.R. Regulação assistencial e a sociedade do conhecimento: o caso do Distrito Federal. Comunicação em Ciências da Saúde, v. 17, n. 4, p. 303-308, 2006.

CONSELHO NACIONAL DE SECRETÁRIOS DE SAÚDE (CONASS). Regulação em Saúde. Coleção Progestores - Para entender a gestão do SUS. Brasília: Conass, 2007. 
DISTRITO FEDERAL (DF). Portaria n 189, de 7/10/2009. Implantação do Complexo Regulador no Distrito Federal. Brasília, 2009. Disponível em: 〈http://www.buriti.df.gov.br〉. Acesso em: 21 abr. 2010.

Plano Diretor de Regionalização do Distrito Federal. Brasília, 2007b. Disponível em: <portal.saude.gov.br/portal/arquivos/pdf/pdr_completo_df.pdf>. Acesso em: 20 mai. 2010.

Portaria $\mathbf{n}^{\mathbf{0}}$ 42, de 31/08/2006. Aprova as Diretrizes e Critérios para admissão e alta em Unidades de Terapia Intensiva na SES/DF. Brasília, 2006a.

Portaria no 41, de 30/08/2006. Cria a Central de Regulação de Internação Hospitalar na Secretaria de Estado de Saúde do Distrito Federal. Brasília, 2006 b.

Ordem de Serviço Conjunta SUPLAN/SAS nº 6, de 12/09/2005. Institui a regulação em dermatologia. Brasília, 2005. Disponível em: 〈www.saude.df.gov.br〉. Acesso em: 13 mar. 2010.

Cartilha de Regulação Assistencial. Brasília: SES/DF, 2004.

DJELLAL, F.; GALLOUJ, F. Mapping innovation dynamics in hospitals. Research Policy, v. 34, p. 817-835, 2005.

Survey of research on health sector innovation. In: WINDRUM, P.; KOCH, P. (Orgs.). Entrepreneurship, Criativity and Management. Cheltenham, UK: Edward Elgar, 2008.

GADREY, J. Emprego, produtividade e avaliação do desempenho dos serviços. In Salerno, M.S. (Org.). Relação de Serviço: produção e avaliação. São Paulo: Senac São Paulo, 2001.

GALLOUJ, Faïz. Innovation in the Service Economy: the new wealth of nations. Cheltenham, UK: Edward Elgar Publishing Limited, 2002.

GALlOUJ, Faïz; WEINSTEIN, Olivier. Innovation in Services. Research Policy, v. 26, p. 537-556, 1997.

GIOVANELLA, L.; LOBATO, L.V.C.; CARVALHO, A.I.; CONILL, E.M.; CUNHA, E.M. Sistemas municipais de saúde e a diretriz da integralidade da atenção: critérios para avaliação. Saúde em Debate, v. 26, n. 60, p. 37-61, 2002

IBANHES, Lauro C.; HEIMANN, Luisa S.; JUNQUEIRA, V.; BOARETTO, Roberta C.; PESSOTO, Umberto C.; CORTIZO, Carlos T.; CASTRO, Iracema E. N.; ROCHA, Jucilene L.; KAYANO, Jorge; LUIZ, Olinda C.; BARBOZA, Renato; TELESI JR., Emílio. Governança e regulação na saúde: desafios para a gestão na Região Metropolitana de São Paulo, Brasil. Cadernos de Saúde Pública, n. 23, v. 3, p. 575-584, 2007.

INSTITUTO BRASILEIRO DE GEOGRAFIA E ESTATÍSTICA (IBGE). Estimativas de População para $1^{\circ}$ de julho de 2009. Diário Oficial da República Federativa do Brasil, DF, 14 ago. 2009.

KOCH, P.; HAUKNES, J. Innovation in the Public Sector. Publin Report n. D20. Oslo: NIFU STEP, 2005. 
LIMA, D. H.; VARGAS, E. R. Estudos internacionais sobre inovação no setor público: como a teoria da inovação em serviços pode contribuir? Revista de Administração Púbilca, v.46, n.2, p.385-401, 2012.

LOCKS, M.T.R. Central de Marcação de Consultas da Grande Florianópolis: um estudo de caso. Dissertação de mestrado, Universidade Federal de Santa Catarina, Florinópolis, 2002.

NASCIMENTO, Adail A. M.; DAMASCENO, Ana K.; SILVA, Maria J.; SALES DA SILVA, Maria V.S.; FEITOSA, Aline R. Regulação em Saúde: aplicabilidade para concretização do Pacto de Gestão do SUS. Cogitare Enfermagem, n. 14, v. 2, 2009.

NELSON, R.; WINTER, S. Uma Teoria Evolucionária da Mudança Econômica. Campinas: Unicamp, 2005.

PIRES, M. R. G. M. et al. Oferta e demanda por média complexidade/SUS: relação com atenção básica. Ciência \& Saúde Coletiva [online]. 2010, vol.15, suppl.1, pp. 1009-1019.

SANTOS, Fausto P.; MERHY, Emerson E. A regulação pública da saúde no Estado brasileiro - uma revisão. Interface (Botucatu), n. 10, v. 19, p. 25-41, 2006.

SANTOS, J. S.; KEMP, R.; SANKARANKUTTY, A. K.; SALGADO JÚNIOR, W.; SOUZA, F. F.; TEIXEIRA, A. C.; ROSA, G. V.; CASTRO-E-SILVA, O. Protocolo clínico e de regulação para o tratamento de icterícia no adulto e idoso: subsídio para as redes assistenciais e o complexo regulador. Acta Cir. Bras. [online], v.23, supl.1, p. 133-142, 2008.

SAVIOTTI, P. P.; METCALFE, J. S. A theoretical approach to the construction of technological output indicators. Research Policy, v.13, i.3, p. 141-151, 1984.

SBICCA, A.; FERNANDES, A. L. A racionalidade em Simon e a Firma Evolucionária de Nelson e Winter: uma visão sistêmica. Anais do XXXIII Encontro Nacional de Economia da Associação Nacional dos Centros de Pós-graduação em Economia, Niterói, RJ, Brasil, 2005.

SCHUMPETER, J. A Teoria do desenvolvimento econômico. São Paulo: Abril Cultural (Coleção Os Economistas), 1982.

WINDRUM, P.; GARCÍA-GONII, M. A neo-Schumpeterian model of health services innovation. Research Policy, v. 37, p. 649-672, 2008.

YIN, Robert. Estudo de caso: planejamento e métodos. Porto Alegre: Bookman, 2010. 